\section{Acute Demyelinating Encephalomyelitis in Malaria}

The recent article on acute demyelinating encephalomyelitis in a patient with falciparum malaria is very interesting [1]. Indeed, the neurological complication of falciparum malaria is not uncommon but the acute demyelinating encephalomyelitis is extremely rare. Recently, a similar case was published in another journal [2]. It was proposed that the pathophysiology is due to immunopathological process [3]. The question is whether the acute demyelinating encephalomyelitis is an exact neurological complication due to falciparum malaria. In the present case, it is no doubt that both malaria and acute demyelinating encephalomyelitis existed. However, the question is whether this is an accidental concomitant occurrence. Some viral infections such as dengue are also endemic in the same setting as malaria [4] and can be the cause of acute demyelinating encephalomyelitis [5]. Since there is no laboratory test to rule out concomitant viral infection, the conclusion can be only detection of two disorders in the patient.

\section{SOMSRI WiwanitKit AND ViroJ WiwanitKI Wiwanitkit House, Bangkhae, Bangkok 10160, Thailand . somsriwiwan@hotmail.com}

\section{REFERENCES}

1. Agrawal A, Goyal S. Acute demyelinating encephalomyelitis in a child following malaria. Indian Pediatr. 2012;49:922-3.

2. Rachita S, Satyasundar M, Mrutunjaya D, Birakishore R. Acute disseminated encephalomyelitis (ADEM)-A rare complication of falciparum malaria. Indian J Pediatr. 2012 Jun 15. [Epub ahead of print]

3. Dey AB, Trikha I, Banerjee M, Jain R, Nagarkar KM. Acute disseminated encephalomyelitis - another cause of post malaria cerebellar ataxia. J Assoc Physician India. 2001;49:756-8.

4. Wiwanitkit V. The importance of accurate diagnosis of dengue fever. Future Virol. 2012;1:53-62.

5. Brito CA, Sobreira S, Cordeiro MT, Lucena-Silva N. Acute disseminated encephalomyelitis in classic dengue. Rev Soc Bras Med Trop. 2007;40:236-8.

\section{Glibenclamide for Neonatal Diabetes}

Neonatal diabetes mellitus, defined as insulin-sensitive hyperglycemia involving the activating mutations in the KCNJ11 gene encoding Kir 6.2, is diagnosed within the first six months of life presenting with glucosuria, polyuria, dehydration, failure to thrive, and diabetic ketoacidosis [1]. Treatment with insulin results in dramatic catch-up growth; insulin may be discontinued in about half these patients, as blood glucose is well-controlled on sulfonylureas (SUs).

We report a case of neonatal diabetes in a 4-month-old baby girl presenting with diabetic ketoacidosis due to p.R201H mutation in the KCNJ11 gene, who was successfully changed from subcutaneous insulin to oral glibenclamide on an outpatient basis.

The patient, who was being treated elsewhere as Type 1 diabetic, had an $\mathrm{HbA1} 1 \mathrm{c}$ of $11.6 \%$ with low serum c-peptide levels $(<0.3 \mathrm{ng} / \mathrm{mL})$ on referral. Since autoimmune diabetes is less common before six months of age, we considered other possible causes of diabetes. While awaiting results of genetic analysis, she was initiated on oral glibenclamide (as a powder dissolved in water) in a dose of $0.3 \mathrm{mg} / \mathrm{kg} / \mathrm{day}$, in three divided doses. Strict self monitoring of blood glucose was done and the insulin was gradually tapered off over next few days. Within four days of starting glibenclamide, repeat serum c-peptide levels done were better $(2.25 \mathrm{ng} / \mathrm{mL})$, indicating increased endogenous production of insulin. Genetic testing of both parents was negative for the mutations.

Presently, the patient is doing well, gaining weight, and is continuing on glibenclamide with a latest $\mathrm{HbAl}$ c of $7.6 \%$ and serum c-peptide level of $2.38 \mathrm{ng} / \mathrm{mL}$. We therefore suggest an outpatient protocol for changing these patients from insulin to Sulfonylureas(SUs) [2].

SUs are introduced gradually over a period of weeks as an outpatient. Glibenclamide dose is increased each week by $0.2 \mathrm{mg}$ per kg per day in divided doses up to a total daily dose of $1.0 \mathrm{mg}$ per $\mathrm{kg}$ per day. It has been found that the pre-breakfast glucose may be very slow to fall and prelunch or pre-evening meal glucose values fall more rapidly and are generally a better marker of response to SUs. In 